\title{
Principles of Embedded Assessment in School-Based Making
}

\author{
Yumiko Muraia*, Yoon Jeon Kimb, Stephanie Changc, and Justin Reichd \\ aFaculty of Education, Simon Fraser University, Burnaby, Canada, @uptownyumiko \\ ${ }_{b}$ Playful Journey Lab, Massachusetts Institute of Technology, Cambridge, U.S.A., @yjkimchee \\ cMaker Education Initiative, Berkeley, U.S.A., @ heyasteph \\ dTeaching Systems Lab, Massachusetts Institute of Technology, Cambridge, U.S.A., @bjfr
}

\begin{abstract}
While there is growing interest among educators in bringing the maker movement into school environments, many schools struggle to closely integrate making into their existing core curriculum, mostly due to the difficulty in assessing learning in maker classrooms. Because of the unique nature of maker-centered learning as a pedagogy, conventional assessment methods often fall short. To address this issue, we conducted a study to design assessment in maker classrooms using a design-based research approach, working closely with middle school maker teachers and coaches. Applying the concept of embedded assessment that is commonly used in digital learning environments into in-person maker classrooms, we explored how assessment that captures diverse learning occur in the process of making. This paper reports on the four design principles of embedded assessment in school-based making that emerged from literature reviews as well as interviews and workshops with the partnering educators. By closely examining the contexts of maker classrooms, we discuss challenges and opportunities for assessment in maker classrooms that can help teachers approach assessment as activities that are seamlessly embedded in the classroom culture, norms, and activities that students are engaged in.
\end{abstract}

\section{Background}

There is a growing interest among educators in bringing the maker movement into school environments (Peppler, Maltese, Keune, Chang, \& Regalla, 2015). Due to maker education's commitment to open exploration and creative ideas, many believe that it can spark and sustain student interest in learning (Clapp, Ross, Ryan, \& Tishman, 2016) and teach necessary STEM knowledge and skills (Sheridan, Halverson, Litts, Brahms, Jacobs-Priebe, \& Owens, 2014). Moreover, many schools who integrate makinghands-on activities that involve activities like digital fabrication, textiles, 3D printing, woodcrafts, or robotics - into their curriculum hope that doing so could support valuable outcomes for future success in the 21st-century global economy (Bybee \& Fuchs, 2006; Florida, 2002; Friedman, 2005), such as development of autonomy (Quinn \& Bell, 2013), problem-solving, sense-making (Martin, 2015), playful attitudes, and a failure-positive mindset (Dougherty, 2013; Martin, 2015). At the same time, many 
school programs struggle to closely integrate making into their existing core curriculum. One of the most common challenges is fitting the inherently iterative, interdisciplinary, and collaborative aspects of making within the existing school structure, especially in regard to assessment and standards (Halverson \& Sheridan, 2014).

A maker-centered approach to education poses multiple challenges for assessment because of its unique characteristics as a pedagogy, and, therefore, conventional assessment methods often fall short in capturing learning and development that occur during making. The first of these unique characteristics is that the making process rarely dictates one right way to accomplish a task because it is often studentdriven, exploratory, and distributed across activities and a group of students.

Assessment in making should afford multiple goals and pathways to completion, and incorporate different ways in which students can express ideas. Second, the exploratory and dynamic nature of maker learning makes it difficult to acquire a comprehensive view of the student learning experience by looking solely at the final product. Instead, it is important to also look at the process of learning: that is, assessment in maker classes should look at how students reach their final products. Third, while assessment is typically used to understand individual performance, maker activities often include collaboration (Clapp, Ross, Ryan, \& Tishman, 2016) where the line between each student's contributions is blurred. Students not only explicitly collaborate on maker projects but also help each other to find resources and materials, share knowledge to troubleshoot, and provide a hand to iterate each other's projects. Assessment in makerbased learning needs to address learning that happens collectively in groups. Fourth, maker activities are dynamic, exploratory, and student-driven, requiring teachers to play the role of facilitator, orchestrating moving pieces in the classroom by supporting different needs of students at different points during a class period. It would be 
logistically impossible to put the burden of data collection purely on teachers. There should be a system where assessment can be addressed collectively between students, teachers, and perhaps other stakeholders to lower the logistical burden. Fifth, maker learning is often inter- and trans-disciplinary, bridging multiple fields of studies such as engineering, art, and science. Even when the main goal is in one discipline, students may need to draw on and build knowledge and skills from other disciplines in order to complete their projects. This makes it difficult to assess specific skills or knowledge in isolation from one another. The assessments used for making need to provide a holistic view of student growth beyond individual disciplines. And finally, the learning and development that can occur in maker classrooms, focused on skills such as agency, problem-solving, and risk-taking, are not easily measured by conventional assessment approaches, and there is as yet no method to satisfactorily measure those skills (Duckworth \& Yeager, 2015). Assessment for making must explore this uncharted territory by looking deeper into how to capture learning in these difficult-to-measure areas.

Given the increased implementation of maker education in school environments, there is a pressing need for a new type of assessment that will enable maker education to take root in school environments. In this study, we investigate what assessment in a maker-centered classroom could look like through the co-design of assessment tools with middle school teachers in two different school districts. Because different maker classrooms have structural limitations and needs that require separate consideration of what would work in their different environments, we identified values, needs, and challenges teachers are experiencing with regards to assessment through interviews and design workshops, yielding essential principles for assessment in making. 
In the following sections, we first review a variety of alternative assessment approaches that are commonly used for open-ended student work to identify the strengths and limitations of those approaches and highlight aspects that might inform the design of assessment for maker learning. We then describe the methods employed to identify design principles. After sharing a brief case study from one of the implementations to show what the principles might look like in real contexts, we will describe each design principle in details, referring to the results from the analysis of interviews with teachers and workshop observations. Finally, we will discuss the remaining questions and limitations as well as future directions.

\section{Review of Alternative Assessment Approaches}

Maker educators have already been experimenting with different assessment methods in their classrooms. For example, during our preliminary interviews with four maker educators in the U.S., the maker educators mentioned documentation tools—-such as journals, conversations, interviews, and surveys - that allow students to communicate their learning and capture them in the process, rather than just as part of the final products. Some educators said that they use the data to give feedback or identify ways to support students; others said that they use the documentation tools to support students' independent learning, by helping them become aware of their own learning and progress. These teachers' practices show that there is no established strategy to assess learning in maker-centered education; rather, each of them is exploring on their own. What these maker educators are exploring are related to the practices of assessment in other disciplines that share characteristics with maker education: primarily, hands-on, project-based, and student-centered learning.

Engineering education, for example, faces similar challenges as maker-centered learning. With a long history of assessing complex student development in not only 
knowledge acquisition but also skills and the application of knowledge (Rompelman, 2000), engineering education commonly uses authentic problems as a way to assess student learning, where students are given scenarios for which they need to develop solutions using specific knowledge and skills. This method, commonly referred to as performance-based assessment, has also been used broadly in the science and mathematics education community. While the issues of operational costs (e.g., time constraints) and difficulty of scoring complex performances to identify complex constructs are ongoing challenges (Davey, Ferrara, Holland, Shevelson, Webb, Wise, 2015), engineering skills and other competencies that require students to generate products or performances have increasingly been valued (Fields, 2014). This approach to assessment — incorporating performance or products of student as evidence of student learning — aligns with the qualities of maker-centered learning, where students are constantly engaged in activities that require application of their understanding of the related knowledge and skills while generating artifacts.

In art education, teachers have explored alternative ways of assessing the process of learning instead of the final product. Although historically there was limited interest in assessing student learning in art education (Gruber \& Hobbs, 2002), portfolios are a commonly employed method of collecting, organizing, and reviewing artifacts created by learners (Dorn, Madeja, \& Sabol, 2004; Gardner, 1989). Many maker educators have adapted portfolios into their assessment process, often by utilizing digital media (Chang, Keune, Peppler, \& Regalla, 2014). While portfolios provide opportunities to shed light on an individual's creative process, the challenge remains of how best to capture meaningful evidence in the moment of making and how to then make sense of the evidence. Instead of using portfolios, other tools such as design journals and diaries could be used to support the rich documentation of objects 
created by makers and to record reflections in ways that seem more authentic to their practice and do not disrupt the flow of the overall making activity.

Similarly, early childhood education has a long history of documenting and supporting student-led learning processes, represented in approaches such as the Reggio Emilia approach that originated in Italy. The Reggio Emilia approach is designed to support and nurture each child's innate qualities and interests through extensive observation and documentation. This process is followed by a collaborative inquiry among a team of educators and parents to analyze, interpret, and understand children's beliefs about the topics being investigated, in addition to their qualities, strengths, needs, and interests (Forman \& Fyfe, 1998). Educators collect various forms of documentation, such as notes, transcripts, slides, photos, and videos, throughout the day. This documentation is also shared with children for reflection and self-assessment. Teachers formulate explanations to describe how children are learning, then design learning experiences based on those hypotheses (Fyfe, 2012). Educators at Reggio Emilia schools do not use any pre-existing assessment frameworks, because they believe the use of such tools would inhibit them from gaining a full understanding of the learning process of children (Dahlberg, Moss, \& Pence, 1999). Although Reggio Emilia programs are unique and differ from most of the schools that this project targets in a number of ways, useful strategies can be taken from their approach. For instance, the process of having several educators, parents, and students work together to understand and interpret documented evidence for learning through extensive inquiries to gain a shared understanding of learning processes can be a useful strategy for the design of assessment tools for maker classrooms.

The approaches outlined above in engineering, art, and early childhood education provide useful insights that maker education can consider to capture rich data 
about learning in hands-on, project-based, and learner-centered environments for assessment. However, challenges remain, such as the logistical difficulty of collecting high quality process-oriented data in the iterative processes, and how to connect the data that shows learning in the process to the assessment of student competencies, needs, and potential for growth.

\section{Embedded Assessment}

In this study, we applied the theory of embedded assessment to explore a new assessment strategy that is suitable for maker-centered classrooms. Embedded assessment is an assessment directly woven into the learning environment and activities so that student learning can be monitored and supported in real-time without interrupting the flow of learning (Shute, Ventura, Bauer, 2009; Wilson \& Sloane, 2000). The approach has been widely adopted in fields such as simulations and video games to design tasks within a system that can elicit evidence of desired outcomes, and to automatically and rapidly capture and process rich data generated during performance. While embedded assessment in digital environments could be applicable to makercentered classrooms, maker classrooms do not have the same affordances as a digital game environments, as data cannot be automatically collected and processed without interrupting the flow of the activity. Therefore, we explored the question of what assessment for maker education could look like, building on to the model of embedded assessment by investigating what can be applied, what needs to be modified, and what needs to be added.

\section{Methodology}

This study took a design-based research (DBR) method to explore assessment for maker classrooms. DBR is a research approach to improve educational practices through an 
iterative process of designing, developing, implementing, and analyzing an intervention while allowing researchers to create generalizable theories of learning or design principles (Brown, 1992; Collins, 1992). DBR is appropriate for this work as it allows researchers to iterate on the design of a new form of assessment with teachers as codesigners and to investigate its relevance in real contexts (Roschelle \& Penuel, 2006). Therefore, DBR allowed the research team to investigate design theories for maker assessment while simultaneously developing and iterating assessment tools. There are three main phases in DBR: preparing the experiment, designing the experiment, and retrospective analysis (Gravemeijer \& Cobb, 2009). This paper reports on the four design principles that emerged out of the first phase of the DBR, where we investigated the problem space for an assessment toolkit through a literature review and interactions with a group of teachers and coaches. We specifically explored the assessment practices teachers had already been conducting, their values and beliefs about assessment, and the challenges they are facing.

\section{Research Sites and Participants}

Throughout this study, we worked with teachers and coaches from two middle schools at two school districts in the U.S. where they have actively integrated maker learning approaches into their curriculum. The schools were selected from a network of schools the research team has worked with in the past. These two schools had distinct characteristics from one another; one school had relatively traditional curriculum and assessment practices, while the other school had implemented a progressive learning and teaching program. The contrasting nature of the collaborating schools allowed us to gain broader perspectives about the assessment contexts teachers face.

One school was located in California and was one of two schools in the small district with 340 students ranging from fourth to eighth grade. The school started a 
specialized STEM program six years ago and has been integrating a maker-centered learning approach ever since. A full-time design innovation coach runs a makerspace at the school, working with other teachers to help integrate a maker learning approach into their core curriculum, while also running extracurricular maker projects and a maker club. In addition, a director of learning and innovation in the district works closely with the coach and teachers to integrate technology and making into their curriculum. From this school, two teachers - a math and science teacher who teaches seventh and eighth graders and an English teacher teaching sixth and seventh graders - as well as a design innovation coach, participated in the study. We also worked with two district leaders to gain more understanding of the school and to support teachers in exploring assessment as part of the research project.

The other school was located in Virginia and was one of six middle schools in a medium-sized district. It was a small "lab" school employing an art-intensive curriculum, working with fewer than 50 students ranging from sixth through eighth grade. The district has been evolving towards more progressive school curriculum that emphasizes mastery learning and student choice while deemphasizing standards-based learning. This particular school started a fully project-based and interdisciplinary curriculum during our research collaboration, having teachers co-teach all students regardless of their specialization and in class periods of long durations. The projects incorporate some form of maker activities, and students often move around in the space depending on their needs for a particular project. Teachers are given considerable autonomy on how to teach their courses, which grants them the flexibility to implement unique curricula. A total of six teachers from this school participated: two science teachers, one math teacher, one technology teacher, one art teacher, and one special education teacher. One science teacher left the study after the first workshop as she was 
transferred to a different school. In addition to the teachers, the school principal and assistant principal, two teaching assistants, and one curriculum coach shared their experiences and supported the implementation of the assessment for this study.

We conducted two 2-day design workshops at the school in California and three 1- to 2-day design workshops at the school in Virginia between April and November 2018. The first workshop at each school focused on building mutual understanding around ongoing assessment practices while also prototyping how our assessment approach could be integrated into their existing maker curriculum and practices. The second and third workshops focused on collecting feedback from the teachers about the early prototypes of tools while preparing for field testing in their classrooms. The tools were tested in small groups, and the majority of time was spent on co-planning and codesigning ways for the tools to be implemented as part of the curriculum and activities planned for the upcoming school year.

\section{Data}

The primary data collected and examined for this first phase were the pre-workshop interviews with teachers, coaches, teaching assistants, and principals from the two schools. A few examples of interview questions include: How do you know good learning is happening? How do you involve students in the assessment process? What is a good assessment for you? And/or what counts as assessment? The interviews were audio-recorded and transcribed for analysis. We also took notes based on observations during the workshops and debrief meetings that were conducted at the end of each day use them as part of the data.

\section{Procedures}

This study was conducted in three main stages. The first stage was a preliminary study 
of ongoing assessment work in schools from the literature review and from each partner school. The pre-workshop interviews conducted for this purpose were coded in two cycles (Saldaña, 2015), descriptive (Miles \& Huberman, 1994) coding and then with pattern coding (Miles \& Huberman, 1994), to identify emerging themes in terms of teachers' ongoing assessment processes as well as the values and challenges teachers perceived. In the second stage, we designed seven assessment tools, building on teachers' insights and our literature review. This design process began in parallel to the first step, incorporating ideas inspired by the understanding established through interacting with the partner teachers. The third stage included testing of those seven tools with partner teachers during the workshops to refine the design. The tools generated through this process were later implemented in classrooms in the second phase of the DBR (which is out of scope for this paper). We conducted debrief meetings with participants each time to gather educator perspectives about assessment in making. Throughout these three stages, the research team held several meetings to identify and iterate on basic principles of assessment in making which emerged from the interactions with teachers and the reviewed literature.

\section{Results and Discussion}

This section introduces four principles of assessment for maker classrooms which emerged out of the literature review as well as analysis of our interactions with teachers and coaches. We first describe one example of our embedded assessment implemented in a middle school classroom to provide general context for the principles. We then elaborate on what we mean by each principle and how we generated these principles with supporting resources. 


\section{Example of embedded assessment in a context}

The partner school in Virginia incorporated embedded assessment into one of their project units using the assessment tools that were developed based on these principles. The theme of the project was board game making, with a goal of building and learning to apply literacy skills and engineering knowledge. This project was about three weeks long, starting with story writing, continuing to designing the game mechanics and building the board game, and concluding with writing down clear instructions to help people learn how to play the game. Students were required to conduct multiple playtesting sessions to get feedback from their peers before the final presentation, which was held in the form of a game night to which family members were invited.

The project consisted of several sub-units to guide the students through the complex process of board game making. Each group was given a binder which teachers pre-filled with the instructions and materials necessary for each unit. At the beginning of each unit, teachers printed one focal construct which was selected out of competencies particularly relevant in maker classrooms and placed it in each group's binder, as a way to remind students (Figure 1). The teachers talked with students about the focal construct at the beginning of each unit. This is the first principle we identified: Assessment of making should be construct-driven. Teachers identified skills and dispositions that are important for the goal of each unit, communicating them upfront with students. Teachers reported that they talked about the constructs on a daily basis, and as a result, how students started to use those constructs to describe their learning process. This connects back to how the next principle-Assessment in making should be seamlessly woven into the culture of the classroom and learning environments - can be implemented in classroom contexts. Placing the focal constructs in places where 
students can see and engage in them every day helps the constructs become part of the classroom culture and therefore helps the assessment be more authentic and embedded.

As students worked on their board games, teachers floated around the groups and gave slips of paper with handwritten notes about any behavior they witnessed that related to the focal construct of the unit. This is a customized version of one of the assessment tools designed from our research project that helps teachers document student performance without interrupting the flow of the classroom or project. The teachers were also using a small camera that produced instant physical photographs, annotating them with notes about the kind of learning represented in the images to create visual documentation of student performance (Figure 2). By providing two different types of evidence of student performance related to the focal construct, teachers successfully produced evidence that could be used to communicate learning outcomes, which we selected as the third principle: Assessment in making should be evidence-centered, generating visible, tangible, and varied forms of evidence for the underlying constructs. Students collected and organized the evidence captured by teachers and as part of the final showcase of their game, presented the folder to display the process behind the making of their board game. In this case, the students did not participate in the process of evidence collection, but they communicated their learning processes using the evidence collected by teachers to parents and to other teachers. This became our final principle: Assessment in making should involve students as active participants in the assessment process.

\section{Principle 1: Assessment of making should be construct-driven}

While making is a process majorly driven by learners, when it comes to assessment of making in schools, the teachers who select a project idea need to initiate 
their assessment process by clarifying what skills, mindsets, or topic areas they intend to foster with the maker activity.

Any assessment design should start with identifying what knowledge, skills, or dispositions we want to assess (Messick, 1994). Since educators are looking for demonstrated evidence for underlying competencies, they need to consider if the students' learning experience will afford abundant opportunities to elicit evidence of their competencies. In practice, however, educators tend to prioritize exploration of project ideas, activities, themes, materials, or tools when they design activity ideas rather than starting with what constructs they value (Wardrip \& Brahms, 2016). Since maker activities often enable learners to produce interesting and professional-looking products without much effort (Blikstein, 2013), both students and teachers focus too much on whether they can perform the tasks at hand while not addressing the fundamental skills and dispositions that the task allows them to develop further.

Coach A (personal communication, April 2, 2018) from the school in California touched upon the importance of creating a space for students to demonstrate their learning through making:

[T]his learner learns best by speaking, so how can we develop an assessment that really can help them (...) versus this student's great at making videos, so how can we develop an assessment strategy to make sure they can demonstrate their understanding through this, and not standardizing it across the board? And I say for all students, because all students have their own learning modalities, and giving them opportunities to demonstrate their understanding of content [is important].

He noted that when a student appears to be underperforming, it does not necessarily mean the student lacks understanding or skills but it could be the case that the activity at hand does not allow multiple ways that the student can demonstrate their competence in the underlying skills. 
This issue became salient when the team worked closely with teachers during a design workshop. For example, a teacher from Virginia had a strong interest in helping develop student agency but her original activity had limited opportunity for students to make their own choices (Teacher D, personal communications, April 12, 2019). With limited time and opportunities, students could not adequately demonstrate agency. After realizing this limitation within the activity in relation to the skills that she intended to foster, she modified the activity design to allow for students to demonstrate how well they make their own decisions. Through these insights, we learned that opportunities for teachers to think critically about the competencies they seek, as they are designing maker activities, can enable them to design assessments that specifically focused on the constructs.

Another rationale of this principle comes from the need for educators to assess broader dimensions of their students' learning beyond content knowledge. Assessment of and focus on the standards are often prioritized over the assessment of the cognitive and non-cognitive skills being developed. Most formal classroom teachers are expected to be accountable for how a maker-centered approach can be used to "teach" certain learning outcomes, especially standards-related content topics. However, the very reason educators implement maker-centered approaches is that the approach allows them to support student learning that is not just about content knowledge but also other skills and dispositions such as agency, collaboration, and risk-taking (Clapp, Ross, Ryan, \& Tishman, 2016).

The interest in assessing broader learning outcomes aligned with themes that emerged from the teacher interviews. In addition to content understanding, teachers provided a variety of learning outcomes that they are looking for in students in relation to making. Agency is one of the most frequently mentioned. Teacher $\mathrm{C}$, who teaches 
science, described that she strives to look for students taking ownership and taking actions using their judgment: "when you see them not wait for my guidance to jump into something, it's really when I know that something's going right" (personal communication, November 5, 2018). Other outcomes include engagement in learning processes and risk-taking.

While teachers have a decent understanding of what these non-standards-based outcomes mean, they often do not have a clear understanding of what evidence of these skills should look like because these skills have not been explicitly addressed in current assessment practices. As a result, communicating learning outcomes with students and other stakeholders becomes a large burden for teachers.

Our examination of conversations with teachers implies that when designing assessment in maker-centered learning, it is important to have teachers start by clearly defining what they expect their students to gain from the learning activities before starting to design the process of learning. The learning outcomes can include skills and dispositions beyond content knowledge, but teachers need to deeply reflect on what assessment might look like in the context of their learning environments.

\section{Principle 2: Assessment in making should be seamlessly woven into the culture of the classroom and learning environments}

In maker-centered learning, it is particularly important that assessment is embedded not only in the activity students are engaged in but also in the classroom norms and culture. Embedded in this context means "opportunities to assess student progress and performance are integrated into the instructional materials and are virtually indistinguishable from the day-to-day classroom activities" (Wilson \& Sloane, 2000).

This principle emerged from conversations with several teachers who mentioned the difficulty of pausing students to ask them to document or reflect on their learning 
experience. One teacher from Virginia explained, "It's hard, even for me, when I'm in the middle of doing something to stop and write a reflection about it" (Teacher A, personal communication, April 12, 2018). She admits the fundamental difficulty of stopping and reflecting while doing something, yet she also mentioned that what is missing in her assessment practice is supporting students in engaging in metacognition, by stopping and reflecting, and going back to what they could not achieve.

One reason it is difficult to stop and reflect during maker-centered learning is that maker-centered learning activities tend to have an immersive and dynamic flow rather than a series of small, linear steps. Pausing while making may require greater cognitive labor in switching back and forth between making and reflection, or may cause a loss of creative momentum. Other similarly immersive learning environments such as educational video games have been working on this problem by utilizing digital environments that can collect performance data stealthily while learners are playing the games without interrupting the learner's activity (Shute, Ventura, Bauer, 2009). Although assessment in in-person maker classrooms cannot be completely stealth, assessment in maker-centered learning environments needs to be embedded into the unique flow of the activity, causing minimal interruption to the process, in order to capture the authentic learning experiences of students.

As the review of assessment in other disciplines illustrated, reflecting during the creative process is not utterly impossible. In art education, for example, critical reflection is historically a part of the learning process where learners reflect on their own thought process and production process while they are engaging in art production (Gardner, 1989). As long as there is a coherent purpose and structure, pausing to document and reflect while making can be a meaningful activity integrated into the process. 
Another reason why reflection might be difficult is that students are often accustomed to talking about their own and each other's learning and progress in a constructive way. One teacher we interviewed was an art educator implementing traditional art critique practices in his maker classroom. He mentioned that students are not used to giving productive feedback to each other:

[For a middle schooler,] getting [them] to talk about their own work, (...) I think it's a little difficult, and it could be dangerous...because they're all about the roasting and burning each other and you can't allow that. So, you have to be very guarded, I think, when you're talking about critiquing work (Teacher B, April 12, 2019).

There can be several potential reasons his students made him concerned about how well they can give feedback to one another's work: students may not have a clear understanding of what qualities they need to look for, they may not have the language to describe their reasoning in a constructive way, or there may not be a norm in the classroom that students, not just a teacher alone, also help one another to produce better work.

Therefore, assessment needs to be woven into not only the learning activities but also the culture of the learning environments, including norms, language, and the behaviors students are used to and comfortable with. By creating a learning experience for such assessment, assessment activities can become accessible, transparent, and adaptable so that students can engage in all aspects of their learning and growth. If there is no culture or habit for constructive assessment practices already in place, it requires new opportunities for students to become familiar with assessment processes and approaches, terminology and vocabulary, tools, and expectations, so that they can participate in the assessment process. 


\section{Principle 3: Assessment in making should be evidence-centered, generating}

visible, tangible, and varied forms of evidence for the underlying constructs

Embedded assessment of maker-centered learning should also be based on several forms of tangible evidence that represent multiple aspects of learning captured throughout the process. This principle is informed by evidence centered design (ECD), a method to make inferences based on evidence about particular competencies or other types of attributes (Mislevy \& Haertel, 2006). ECD enables educators and researchers to aggregate information about a learner's performance from various sources and therefore helps them understand what students can do and how they develop the particular constructs or dispositions. Although making activities are a hands-on exploratory process where students are engaged in a variety of tangible materials, a number of assessments in makerspaces still rely on traditional forms of assessment such as surveys and multiple-choice questions at the end of the activities focusing on engagement and interest (Weiner, Lande, \& Jordan, 2018). Given that maker-centered learning is an exploratory process often incorporating multiple means of learning, assessment in maker environments should be based on rich evidence generated by students and teachers throughout the process of making.

Collecting evidence of learning is not straightforward because many of the learning outcomes in maker classrooms are hard to measure. Maker-centered learning provides students an opportunity to develop a variety of knowledge, skills, and attitudes (Clapp, Ross, Ryan, \& Tishman, 2016). The teachers we interviewed also mentioned various learning outcomes they wish to see through maker activities. However, the connection between what students are making and those constructs are often not visible in classrooms. For example, agency, collaboration, and engagement are the three most frequently mentioned desired learning outcomes from our interviewees, but they are not 
necessarily visible by just looking at what students are making. It requires careful observation of how students are making. One teacher described that she notices and takes mental notes of each student. However, such method rarely works at scale or for novice teachers.

Another reason for the difficulty to collect evidence in maker-centered learning is that it often takes complex and non-linear paths where students explore different materials and tools through a series of trial-and-error and social interaction. Learning activities often involve varied extents of open-endedness where students are given the freedom to decide what to make or how to make it. As a result, each student tends to take a unique and complex approach to achieve the instructions given to them. A single assessment method at the end of a learning activity is often not comprehensive enough to fully capture and understand students' diverse learning experiences.

Teacher G (personal communication, November 5, 2018), who teaches English at the school in California, talked about how difficult it is to understand the students' learning outcomes:

With writing, it's just so straightforward that it's pretty easy [to see] whether or not they're meeting the standards. [But for maker projects,] am I going to have to go through every kid, and take the standards and say, "Okay, did you master this one, did you master this one?" How is that going to look?

While asking each student is one way of gathering evidence, it is a time-consuming approach and also fails to give a complete picture of how students are growing their competencies.

The teachers we interviewed also faced the challenge of sharing the learning outcomes with parents and other stakeholders. One teacher observed, "we as educators can pick out the learning, because we're trained to do that. But, how do you then convey to a parent, who has no experience?" (Teacher F, personal communication, November 
5, 2018). For students, parents, and other stakeholders who without the expertise to understand student performance, it is not easy to gain a comprehensive view about learning outcome. In addition, signs of learning in maker-centered activities are often witnessed in the process of making and it's difficult to capture concrete evidence in the moment. Collecting visible and tangible representation of those indicators in real-time is essential for sharing evidence of learning that is highly time- and place-specific with people who were not there to see it themselves.

Tangible evidence can help students and teachers alike to recall and reiterate the experience more accurately specifically with parents and administrators who are attempting to understand the full scope of what students are learning in maker-centered classrooms. For these reasons, embedded assessment for maker-centered learning needs to help students and teachers generate visible and tangible evidence that can be used to convey learning beyond just "the thing they created." Collecting a variety of visible and tangible pieces of evidence that provide multidimensional perspectives on students' performance at multiple points during maker activities is an alternative approach to capturing such complex learning experiences. For example, written documentation can be combined with photographs or videos to incorporate different mediums; descriptive documentation can be combined with a quantitative record of the number of times certain behaviors happened; or observational notes and reflections by students can be combined with observational notes by teachers. By collecting a variety of types of evidence, both students and teachers gain alternative ways of looking at their performances. This may also open up an opportunity for students who are not competent in one type of medium (such as writing) to express their understanding through different mediums (such as drawing). 


\section{Principle 4: Assessment in making should involve students as active participants in the assessment process}

In maker-centered learning environments, students should be empowered as the assessor of their own learning and choose and collect evidence for their own learning to construct a multidimensional and comprehensive account of their learning experiences. Historically, assessments have been conducted by teachers to and for students in order to assess students' abilities and compare students with one another, often relying on summative assessment after the learning activity. Most of the time a universal measure is used to assess student learning, even though it may not guarantee equal access to learning for students with diverse economic, cultural, and academic backgrounds.

Maker activities involve many exploratory and complex interactions in more or less open-ended environments that can lead to varied outcomes. Not only can the product and learning goals vary between students but also the process taken to reach learning goals can as well. In order to understand what students gained through the activity, assessment needs to capture learning that occurs during the process of making. About half of the teachers described that they wished to have better ways to holistically capture students' learning processes. Teacher A from the school in Virginia mentioned, "we miss a lot when our assessments just show an endpoint, and they don't show the growth experience.” and continued, “"The journey is more important than the destination,' is true in learning, I think, as well” (personal communication, April 12, 2018). She emphasized that she believes that assessment lies in the process of students getting from point A to point B. Another coach mentioned that they focus greatly on reflection on the learning process rather than the project itself, such as lighting up a circuit (Coach B, personal communication, Apr 2, 2018). The importance of looking at the process of maker-centered learning was shared among many interviewees that we 
talked to.

However, observing and collecting learning evidence in the process requires teachers to pay attention to multiple students at the same time, documenting multifaceted and dynamic learning outcomes throughout the class period. This is nearly impossible to achieve, particularly for teachers who do not have the privilege of having another teacher or assistant in the classroom. Empowering students to collect learning evidence and self-assess their progress is, therefore, not only compatible with the values of making, but also a practical solution in maker-centered classrooms.

In addition, involving students in the assessment process can provide an opportunity for students and teachers to create a shared understanding of when and how learning occurs with making. Since the making process is complex and highly contextualized, teachers may be able to capture limited moments of each student in the class. Relying solely on teachers, therefore, eliminates the opportunity for both students and teachers to coherently understand students' full learning experiences.

A number of interviewees answered that one of the qualities of assessment that matters to them was that the assessment helps students understand and be on-board with it. Coach C described his idea of a good assessment:

I think a good assessment is one that students understand why they're doing it, students understand what they're doing, and then what it leads to. What's the next step on this, if there is the next step? Who's the audience of this thing that I'm doing? (personal communication, April 12, 2019)

By having students document their own learning, explaining and communicating their learning experience, students can deepen their understanding about their learning experiences while teachers can also gain more understanding about what students got out of the class activity. 
There are several conditions that are necessary for truly engaging students in the assessment process, as mentioned by a teacher from the school in Virginia: "I think we need a system for having kids capture that [emphasis added]." (Teacher E, personal communication, November 5, 2019). First, students and teachers need to build a shared understanding of what they are assessing. For example, if how students define collaboration differs from how the teacher defines it, they cannot collect consistent evidence that shows how students are collaborating with one another. It is important that students and teachers take time together to share how they understand the construct and agree upon what it might look like in their classroom context.

Second, the series of skills to assess learning-i.e., noticing the signs of learning, documenting it, understanding what it means, and communicating about it with other stakeholders - is not a skill that is universally shared among students. Students need to learn assessment and practice it before they can begin assessing their own learning performance in a real context.

Third, the design of the assessment should be inviting and accessible to students for them to openly and sincerely participate in the assessment process. Teachers, administrators, and researchers tend to use specific words when they talk about assessment that are not necessarily understandable to students, from general assessment terms like construct and disposition to educational terms such as intention and scaffolding. It is important that facilitation of assessment and materials use plain language that students are familiar with, and involve engaging activities for students to take part in.

In sum, we learned from the interaction with teachers that it is important that embedded assessment in making should position students as active and empowered participants in the assessment process, collectively noticing, capturing, understanding 
and communicating learning experiences, paying attention to the conditions described above. This would help not only teachers but also students themselves build deeper understanding of the learning experiences that are taking place.

\section{Discussion}

In this paper, we discussed how we established design principles to create tools that enable embedded assessment in school-based making to better support assessment of students' learning demonstrated in the messy, creative, and iterative process of making. Through literature review and analysis of interviews and workshops with teachers, we identified the gap that exists in how process-oriented evidence is being collected and used to connect with underlying constructs. We then turned to the theory and practice of embedded assessment (e.g. Wilson \& Sloane, 2000) to establish design principles for the assessment of school-based making, while iteratively designing, with teachers as codesigners, assessment tools that embody those principles.

The principles work as a guide for any assessment tools by helping teachers and student envision assessments that are fundamentally different from common assessment tools, such as rubrics and portfolios, to better assess the complex nature of open-ended student work such as maker projects. Although it is not the scope of this paper, we have designed assessment tools based on these principles that are designed to support teachers and students in creating a shared understanding of the maker skills that teachers intend to foster and in assessing those skills during the process of making. Those tools enable students to play an active participant role with evidence collection and meaningmaking with the collected evidence, instead of having assessment being done at or to them.

The current work illuminates design challenges to embedding assessment in non-digital learning environments, particularly with regard to collecting process data. 
By embedding assessment in making, as a context for assessment, this work provides an opportunity to push the boundaries of what role assessment can play in classrooms. For example, in making, the student might modify the goal of an activity as they proceed with making, and there could be multiple dynamic paths to an end state. Also, making is often social, where students build upon each other's ideas and failures collectively, which also contradicts traditional assumptions for assessment.

The present work also demonstrates principles of assessment that can blur the distinction between making (and therefore learning) and assessment. Ultimately, the goal of such an assessment is to foster development of teachers' practices aligned with the pedagogical values of making, as well as to improve overall learning outcomes. Therefore, future research needs to investigate how embedded assessment in making might impact the quality of teaching and learning. That is, how does embedded assessment of making help students understand the kinds of skills that they are developing from maker activities, and how are those skills connected to their interests and goals? Similarly, how does this approach to assessment help teachers design and orchestrate various aspects of the activity to be true to the pedagogical values of making? How does this help teachers to better support student learning? Additionally, because the current set of principles is based on one full design cycle of the designbased research approach (Gravemeijer \& Cobb, 2009) where we created usable tools that are implemented in real contexts, further assessment tool development and iteration will also allow us to expand and iterate these principles, especially in relation to the potential benefits of such an assessment approach. Furthermore, our principles (and therefore our tools) have only been tested within two schools, and, therefore, we will need to continue to investigate the generalizability of both tools and principles in other contexts. 


\section{Conclusion}

When teachers integrate making into their classrooms, they want students to benefit from the inherent values of making, such as agency, iteration, and social scaffolding. However, when making is being fit into the school structure, it begs the question of how assessment needs to be conducted in order to capture growth and development with regard to those values. Some of the existing practices, such as portfolios and rubrics, are useful, but they are limited when it comes to capturing rapidly generated evidence in the moment of making. As a result, assessment of making instead tends to focus on the end products (e.g. a showcase). The assessment principles described here can help teachers approach assessment as activities that are seamlessly embedded in the classroom culture, norms, and activities that students are engaged in. It is challenging for individual teachers to create assessment tools that serve this purpose. Therefore, more research needs to be done exploring scaffolding for teachers to understand assessment and assessment tools, modify them to meet their needs, and implement assessments that address the teachers' desires related to better assessment in making.

\section{References}

Blikstein, P. (2013). Digital fabrication and 'making' in education: The democratization of invention. FabLabs: Of machines, makers and inventors, 4, 1-21.

Brown, A. L. (1992). Design experiments: Theoretical and methodological challenges in creating complex interventions in classroom settings. The journal of the learning sciences, 2(2), 141-178

Bybee, R. W., \& Fuchs, B. (2006). Preparing the 21st century workforce: A new reform in science and technology education. Journal of Research in Science Teaching: The Official Journal of the National Association for Research in Science Teaching, 43(4), 349-352.

Chang, S., Keune, A., Peppler, K., Maltese, A., McKay, C., \& Regalla, L. (2015). Open portfolios: Maker education initiative full research brief series. 
Clapp, E. P., Ross, J., Ryan, J. O., \& Tishman, S. (2016). Maker-centered learning: Empowering young people to shape their worlds. John Wiley \& Sons.

Collins, A. (1992). Toward a design science of education. In New directions in educational technology (pp. 15-22). Springer, Berlin, Heidelberg.

Dahlberg, G., Moss, P., \& Pence, A. R. (1999). Beyond quality in early childhood education and care: Postmodern perspectives. Psychology Press.

Davey, T., Ferrara, S., Shavelson, R., Holland, P., Webb, N., \& Wise, L. (2015). Psychometric considerations for the next generation of performance assessment. Washington, DC: Center for K-12 Assessment \& Performance Management, Educational Testing Service.

Dorn, C. M., Sabol, R., Madeja, S. S., \& Sabol, F. R. (2014). Assessing expressive learning: A practical guide for teacher-directed authentic assessment in K-12 visual arts education. Routledge.

Dougherty, D. (2013). The maker mindset. In Design, make, play (pp. 25-29). London, UK: Routledge.

Duckworth, A. L., \& Yeager, D. S. (2015). Measurement matters: Assessing personal qualities other than cognitive ability for educational purposes. Educational Researcher, 44(4), 237-251.

Fields, R. (2014). Towards the National Assessment of Educational Progress (NAEP) as an Indicator of Academic Preparedness for College and Job Training. National Assessment Governing Board.

Florida, R. (2002). The rise of the creative class and how it's transforming work, leisure, community and everyday life. New York, NY: Basic Books.

Forman, G., \& Fyfe, B. (1998). Design, documentation and discourse: A theory of negotiated learning. The hundred languages of children: The Reggio Emilia approach to early childhood education (2nd edition). Greenwich, CT: Ablex Publishing.

Friedman, T. (2005). The world is flat: a brief history of the globalised world in the 21st century. London, UK: Allen Lane-Penguin Books.

Fyfe, B. (2012). The relationship between documentation and assessment. The hundred languages of children: The Reggio Emilia experience in transformation, 3, 273291.

Gardner, H. (1989). Zero-based art education. Studies in Art Education: A Journal of Issues and Research, 30(2). 
Gravemeijer, K., \& Cobb, P. (2006). Design research from a learning design perspective. In Educational design research (pp. 29-63). Routledge.

Gruber, D. D., \& Hobbs, J. A. (2002). Historical analysis of assessment in art education.

Halverson, E. R., \& Sheridan, K. (2014). The maker movement in education. Harvard educational review, 84(4), 495-504.

Martin, L. (2015). The promise of the maker movement for education. Journal of PreCollege Engineering Education Research (J-PEER), 5(1), 4.

Messick, S. (1994). The validity of psychological assessment: Validation of inferences from a person's responses and performances as scientific inquiry into score meaning. ETS Research Report Series, 1994(2), i-28.

Miles, M. B., \& Huberman, A. M. (1994). Qualitative data analysis: An expanded sourcebook (2nd ed.). Thousand Oaks, CA: Sage.

Mislevy, R. J., \& Haertel, G. D. (2006). Implications of evidence-centered design for educational testing. Educational Measurement: Issues and Practice, 25(4), 6-20.

Peppler, K., Maltese, A., Keune, A., Chang, S., \& Regalla, L. (2015). The maker ed open portfolio project: Survey of Makerspaces, Part II. Open Portfolios. Berkeley, CA: Maker Ed.

Quinn, H., \& Bell, P. (2013). How designing, making, and playing relate to the learning goals of K-12 science education. In Design, Make, Play (pp. 35-51). Routledge.

Rompelman, O. (2000). Assessment of student learning: evolution of objectives in engineering education and the consequences for assessment. European journal of engineering education, 25(4), 339-350.

Saldaña, J. (2015). The coding manual for qualitative researchers. Sage.

Sheridan, K., Halverson, E. R., Litts, B., Brahms, L., Jacobs-Priebe, L., \& Owens, T. (2014). Learning in the making: A comparative case study of three makerspaces. Harvard Educational Review, 84(4), 505-531.

Shute, V. J., Ventura, M., Bauer, M., \& Zapata-Rivera, D. (2009). Melding the power of serious games and embedded assessment to monitor and foster learning. Serious games: Mechanisms and effects, 2, 295-321.

Wardrip, P. S., \& Brahms, L. (2016). Taking making to school. Makeology: Makerspaces as learning environments, 1, 97-106.

Weiner, S., Lande, M., \& Jordan, S. S. (2018). What Have We" Learned" from Maker Education Research? A Learning Sciences-base Review of ASEE Literature on 
the Maker Movement. Review \& directory-American Society for Engineering Education.

Wilson, M., \& Sloane, K. (2000). From principles to practice: An embedded assessment system. Applied measurement in education, 13(2), 181-208. 


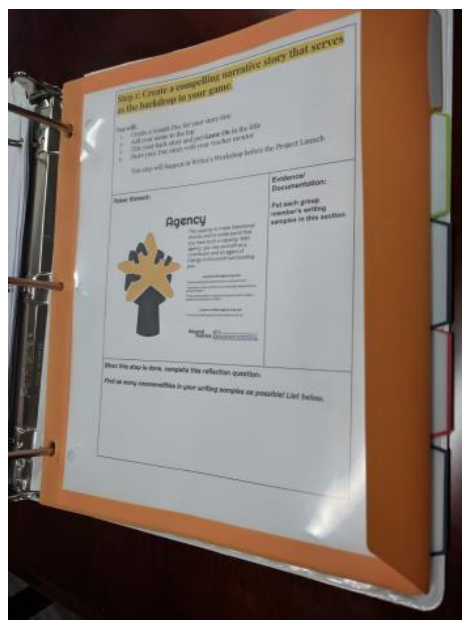

Figure 1. Maker construct on the cover of the unit.

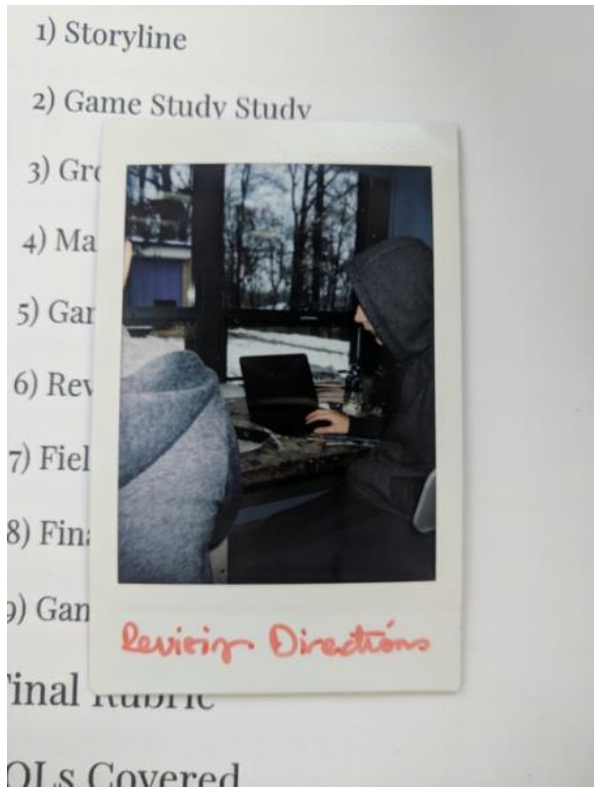

Figure 2. Visual documentation with notes regarding how this student displayed design process skills, which was one of the constructs featured during this project. 